\title{
nature
}

\section{The importance of coherent defence strategies}

Astronomers served by the European Space Agency run the risk of undermining their own future by undue criticism of it, and by underestimating the obstacles to imitating NASA.

urope's particle physicists can face the future with a fair degree of satisfaction. Following recent international endorsements of CERN, they are set to occupy centre stage in the next generation of facilities at the high-energy frontiers of their discipline. European astronomers, in contrast, are rightly worried about their future. In particular, as a result of the downward pressure on budgets, those depending on space platforms find themselves in a new and insecure world where they can no longer rely on the European Space Agency (ESA) to provide them with a nourishing torrent of data. But whether, as some claim, their future can be much invigorated by following the example of the US National Aeronautics and Space Administration (NASA) is doubtful.

ESA's Horizons 2000 programme has provided a relatively secure environment for astronomers, combining stability, through regular launches of large cornerstone missions, with flexibility, through intermittent medium-sized missions which are open to competition from the research community over significantly shorter timescales. The ability of ESA to plan reliably for the future has long been envied by US astronomers. But ESA's member states are no longer able to pay for the visions approved in the $1980 \mathrm{~s}$, and rethinking is required. The first report to emerge, led by ESA's Space Science Advisory Committee (SSAC), proposes maintaining the Horizons 2000 missions more or less as they are, but stretching out their launch schedules.

A prospect of periods of data starvation is causing agitation among lastronomers in Europe. And that may be the reaction that SSAC is hoping for, acting as it is from a genuine sense of gloom but also, no doubt, in a spirit of political agitation. If the SSAC is hoping that such worries will cut much ice with research ministers from ESA's member states, it is deluding itself. More productively, however, its preliminary recommendations will concentrate ESA's mind on the necessity to question all traditional assumptions in order ruthlessly to cut costs.
Even more pressure in that direction comes from Germany, France and Britain, whose governments, and some of whose astronomers, are calling aggressively for ESA to take lessons from NASA's streamlining of its programmes according to the slogan "smaller, faster, cheaper, better". It is the obvious route to healthy science and healthy balance sheets, say ESA's critics, and if NASA can do it, so can Europe.

Such words are cheap but can also be powerful, and they need to be used carefully when governments have their budget knives sharpened. NASA's approach has not yet been tested - its programme has been held up by launcher problems. Furthermore, the need to develop technologies to deliver efficient, fast, cost-effective science puts new costs up front - NASA's ability to develop them cost effectively has yet to be demonstrated. But NASA has benefited greatly from its inheritance of declassified technologies in cryogenics, detectors, adaptive optics and the like, to which ESA does not have access. And the strong radical leadership possible at NASA cannot readily emerge within ESA, bound up as it is in the inescapable Euro-paraphernalia of negotiations around member-state interests.

To its credit, ESA is reducing several components of its inertia, albeit belatedly. Its director of science, Roger Bonnet, has set up task forces that will report to the SSAC at the end of this month, to investigate in detail how NASA is introducing cost-savings and shortening its time to launch, and deciding which ideas can be imported. Individual missions and organizational procedures are again being scrutinized for cost savings. For these reasons, the SSAC's next report will no doubt be more trenchant about changes to be made within ESA, ahead of the crucial decisions about the Horizons programme's future to be made later this year. In the meantime, critics of ESA who nevertheless wish it well should choose their words carefully. CERN's success at surviving member-state pressures has been as much a result of the coherence among its research community as of anything else.

\section{Will Japan's money for science be short lived?} Japan's scientists should take note of criticism of the Japan Key Technology Centre.

apanese scientists, long seen as poor cousins of their Western counterparts, have been enjoying an unprecedented bonanza of extra government funding in recent years. And, if the five-year plan mapped out by the government last year is to be believed, the money should continue to flow liberally for quite some time.

But the first signs of a backlash against the extra money for science may be appearing. In recent weeks, Japan Key-TEC, an organization that was set up with income derived from privatization of Nippon Telegraph and Telephone Corporation, has come under criticism for failing to deliver a significant return on more than $¥ 200$ billion in investment over the past ten years (see page 424).

Proponents of Japan Key-TEC argue that it is unreasonable to expect an immediate return on the basic research that has been abundantly funded by the centre at institutes such as the Biomolecular
Engineering Research Institute (BERI) in Osaka. That is true. But BERI is an exception among Japan Key-TEC institutes. Most of the centre's 60 or more institutes have made little or no impact on the world of science or technology and are being quietly closed down.

Japan's government and university scientists are likely to face similar criticism a few years hence if they fail to produce visible results. The science community must act now to ensure that, through rigorous peer review, the extra money goes to deserving scientists and that particular individuals in priority fields do not get a surfeit of funds. Furthermore, greater efforts must be made to publicize the achievements of institutions and individuals through, on the one hand, objective external reviews and assessment and, on the other, media releases that explain the science to the general public. Otherwise, Japan's scientists may soon become poor cousins again. 